\title{
Design of a CMOS closed-loop system with applications to bio-impedance measurements
}

\author{
Alberto Yúfera, Adoración Rueda
}

Instituto de Microelectrónica de Sevilla (IMS), Centro Nacional de Microelectrónica (CNM-CSIC) Universidad de Sevilla, Av. Américo Vespucio s/n. 41092, Sevilla, Spain

Keywords:

Impedance measurements

Electrical bio-impedance

Electronic instrumentation

Biometric circuits

CMOS analog circuits

Sensory systems

\begin{abstract}
A B S T R A C T
This paper proposes a method for impedance measurements based on a closed-loop implementation of CMOS circuits. The proposed system has been conceived for alternate current excited systems, performing simultaneously driving and measuring functions, thanks to feedback. The system delivers magnitude and phase signals independently, which can be optimized separately, and can be applied to any kind of load (resistive and capacitive). Design specifications for CMOS circuit blocks and trade-offs for system accuracy and loop stability have been derived. Electrical simulation results obtained for several loads agree with the theory, enabling the proposed method to any impedance measurement problem, in special, to bio-setups including electrodes.
\end{abstract}

\section{Introduction}

Impedance is a useful parameter for determining the properties of matter [1]. Today, many research goals are focused to measure the impedance of biological samples. There are several major benefits of measuring impedances in medical and biological environments: first, most biological parameters and processes can be monitored using its impedance as marker [2-5]. Second, bioimpedance measurement is a non-invasive technique and, third, it represents a relatively cheap technique in labs. Impedance Spectroscopy (IS) in cell cultures [6] and Electrical Impedance Tomography (EIT) in bodies [7] are examples of the impedance utility in this field.

For the problem of measuring a given impedance $Z_{x}$, with magnitude $Z_{x o}$ and phase $\phi$, several methods have been reported. Commonly, these methods require excitation and processing circuits. Excitation is usually done with Alternating Current (AC) sources, while processing steps are based on coherent demodulation principle [1] or synchronous sampling [8]. In both, processing circuits must be synchronized with excitation signals, as a requirement for the technique works, obtaining the best noise performance when proper filter functions (High-Pass (HP) and Low-Pass (LP)) are incorporated. Block diagrams for both are

\footnotetext{
* Corresponding author.

E-mail addresses: yufera@imse-cnm.csic.es (A. Yúfera), rueda@imse-cnm.csic.es (A. Rueda).
}

illustrated in Figs. 1 (a) and (b), respectively. The main drawback for the Ackmann method [1] is that the separated channels for in-phase and quadrature components must be matched to avoid large phase errors. Synchronous sampling proposed by Pallás avoids two channels and demodulation, by selecting accurate sampling times, and adding an HP filter in the signal path to prevent low-frequency noise and sampler interferences. Both work as feed-forward systems: the signal generated on $Z_{x}$ is amplified and then processed. The setups for bio-impedance measurements usually include electrodes between their components as sensor interface between the electronic instrumentation and the bio-samples, so when excitation signals are applied, the electrodes' performance could be considered as part of the load is being excited from driving circuits point of view. A detailed description of the electrode models for biological measurement can be found in [5]. The presented work proposes a closed-loop method for bio-impedance measurement based on the AC voltage source application, with constant amplitude, to impedance under test (ZUT). This method can be applied to electrode-based sensor systems, solving the main problems of using electrodes and their impedance frequency dependence in the following forms: first, limiting by design the voltage applied to electrodes. This allows biasing the electrodes at the linear operation region. Second, it permits incorporating the frequency dependence of the electrode impedance to design equations, making easier the selection of the working frequency and allowing the optimization of the system performance, since it could be possible to set the frequency for optimum system 
response. Once these electrode constrains are considered, the proposed circuits deliver magnitude and phase impedance signals directly in easy from to be acquired: a time constant voltage, for magnitude, and the duty cycle of a digital signal, for phase.

This paper in organized as follows: Section 2 describes the proposed CMOS closed-loop system for impedance measurement. In Section 3, the main circuits employed for impedance measurement are reported. Design system considerations for loop stability are given in Section 4. Simulation results for several types of loads and setups will prove the correct performance of the proposed system in Section 5. Conclusions are underlined in Section 6.

\section{Proposed impedance measure system}

The proposed circuits for the measurement of impedance magnitude, $Z_{x o}$, consider an AC current excitation signal, with $\omega$ frequency. The circuits are designed to work maintaining a constant amplitude across the load $\left(V_{x o}=\right.$ cte), known as Potentiostat (Pstat) condition. The proposed circuit block diagram, shown in Fig. 2, has the following as main components: an Instrumentation Amplifier (IA), a rectifier, an error amplifier, and a current oscillator with programmable output current amplitude. The voltage gain of the instrumentation amplifier passband is $\alpha_{i a}$. The rectifier works as a full wave peak-detector,

a

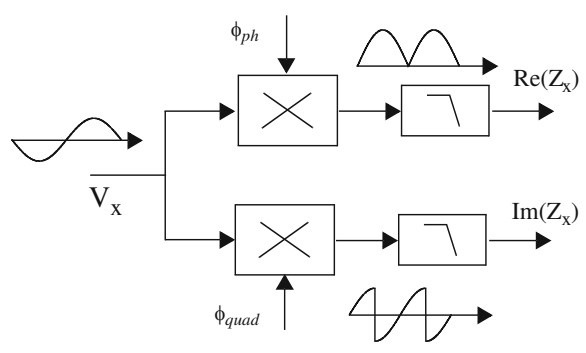

b

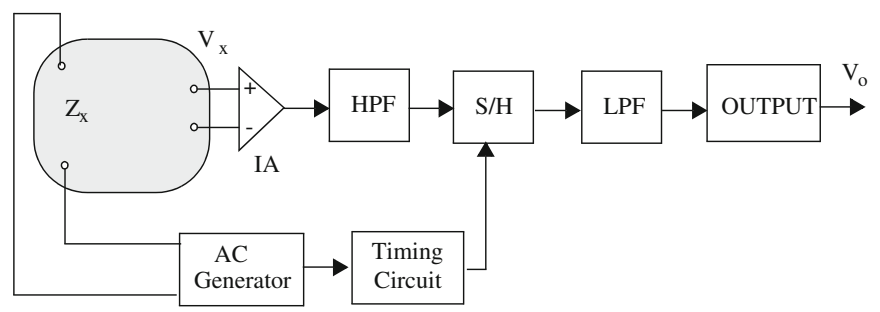

Fig. 1. (a) Coherent demodulation. (b) Synchronous sampling. sensing the $V_{o}$ peak-to-peak voltage value. Its output is a time constant voltage, $V_{d c}$, with $\alpha_{d c}$ gain $\left(V_{d c}=\alpha_{d c} \alpha_{i a} V_{x o}\right)$. The error amplifier, with $\alpha_{e a}$ gain, will compare the DC signal with a reference, $V_{r e f}$, to amplify the difference. The current oscillator generates the AC current to excite the load. It is composed of an external AC voltage source, $V_{s}$, an Operational Transconductance Amplifier (OTA) with $g_{m}$ transconductance, and a four-quadrant voltage multiplier with $K$ constant. The voltage generated by $V_{s}$, $V_{s o}, \sin \omega t$, is multiplied by $V_{m}$, and current converted by the OTA. The equivalent transconductance from the magnitude voltage signal, $V_{m}$, to the excitation current, $i_{x}$, is $G_{m}=g_{m} \cdot V_{s o} \cdot K$. A simple analysis of the full system gives the approximated expression for the voltage amplitude at $V_{x}$

$V_{x o}=\frac{V_{r e f}}{\alpha_{i a} \cdot \alpha_{d c}}$

when condition

$Z_{x o} G_{m} \alpha_{e a} \alpha_{i a} \alpha_{d c} \gg 1$

is satisfied. It is defined the system closed-loop gain as $\alpha_{o}=Z_{x o} G_{m} \alpha_{i a} \alpha_{d c} \alpha_{e a}$. Voltage in Eq. (1) remains constant if $\alpha_{i a}$ and $\alpha_{d c}$ remain constant too. Hence, Pstat condition is fulfilled if condition in Eq. (2) is true. Considering the relationship between the current $i_{x}$ and the magnitude voltage $V_{m}\left(i_{x o}=G_{m}, V_{m}\right)$, the impedance magnitude is

$Z_{x o}=\frac{V_{x o}}{G_{m}} \cdot \frac{1}{V_{m}}$

Eq. (3) shows that from voltage $V_{m}$, the impedance magnitude $Z_{x o}$ can be calculated, since $V_{x o}$ and $G_{m}$ are known from Eq. (1) and the design parameters. The impedance phase could also be measured with $V_{\phi}$ signal in Fig. 2, by considering the input voltage oscillator, $V_{s}$, in phase with the $i_{x}$ current. This signal can be squared or converted into a voltage digital signal, to be used as time reference or sync signal $\left(V_{x d}\right)$. The $V_{o}$ voltage is also converted into a squared waveform $\left(V_{o d}\right)$ by means of a voltage comparator. If these two signals feed the input of an EXOR gate, a digital signal will be obtained, $V_{\phi}$, called phase voltage, whose duty cycle, $\delta$, is directly proportional to the phase to be measured.

From Eq. (2), the range of $Z_{x o}$ magnitude value must be known in order to be satisfied. However, depending of the set-up for measuring employed, the $Z_{x o}$ can include different parasitics. In four-wire based systems, these effects are minimized by the high input impedance amplifier, but in two-wire ones, the amplifier output voltage delivers the contribution of both impedances, namely from the set-up and ZUT, so $Z_{x o}$ must be known and quoted before to define the circuit specifications. Fig. 2 illustrates a two-wire system. The impedance to be measured should be placed between electrodes $e_{1}$ and $e_{2}$.

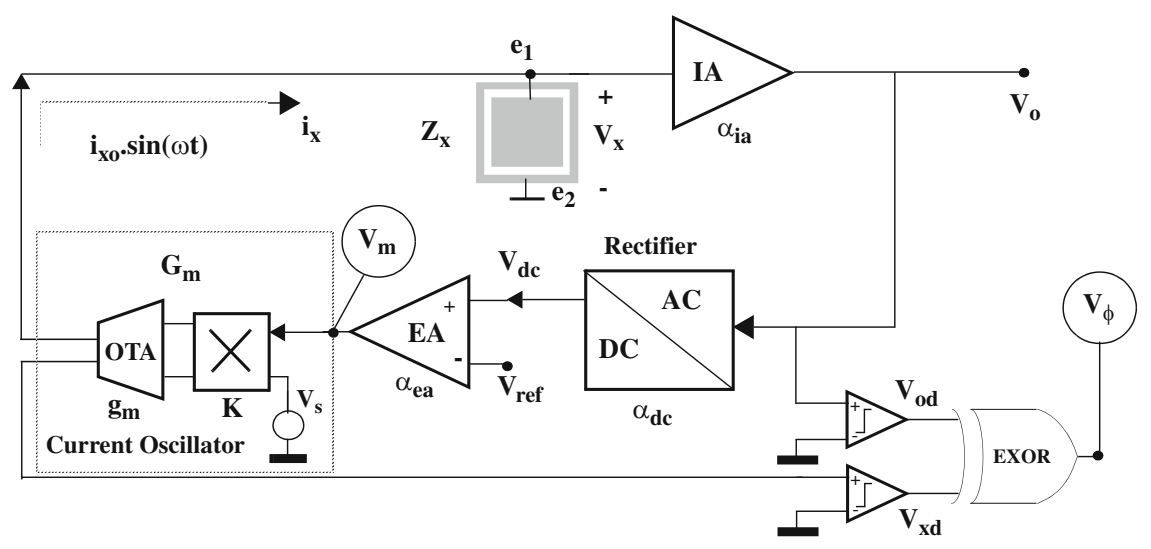

Fig. 2. Proposed circuit blocks for impedance sensing. Magnitude and phase are obtained from signals $V_{m}$ and $V_{\phi}$, respectively. 
The amplitude of the input voltage instrumentation amplifier, $V_{x o}$, can be now limited, thanks to the feedback loop, enabling the proposed system for impedance measures based on electrodes [5], as is the case of bio-impedance measure systems [9]. Changes with frequency in magnitude of electrode impedances can be incorporated to $Z_{x o}$ in order to satisfy Eq. (2).

Absolute errors at impedance magnitude measurements will be dependent on circuit parameters $\alpha_{i a}, \alpha_{d c}$ and $G_{m}$, as expressed in Eqs. (1) and (3). These error sources are similar and common for other impedance techniques [1,2]. Also, phase errors have an additive contribution from the instrumentation amplifier phase response, which have to be considered. In many applications, the relative impedance changes, evaluated after system calibration, contain the most relevant information and relax the circuit performance specifications. Errors in the aforementioned parameters could limit the system performance.

\section{CMOS circuit design}

The circuits required to implement the blocks in Fig. 2 have been designed in a $0.35 \mu \mathrm{m}$ CMOS technology for $3 \mathrm{~V}$ power supply. Design parameters were adjusted initially for $10 \mathrm{kHz}$ frequency, with $Z_{x o}=100 \mathrm{k} \Omega$. The parameters chosen were $\alpha_{o}=100, \alpha_{i a}=10, \alpha_{d c}=0.25, \alpha_{e a}=500, G_{m}=1.2 \mathrm{uS}$, and $V_{r e f}=20 \mathrm{mV}$. The $Z_{x o}$ value can belong only to impedance to be measured or include also the set-up contribution. In all cases, its minimum value must be known to fulfil Eq. (2).

\subsection{Instrumentation amplifier}

The instrumentation amplifier circuit schematic is shown in Fig. 3. It is a two-stage amplifier: a transconductance input stage and a trans-resistance output stage, where filtering functionality has been included. The passband frequency edges were designed according to the frequency range common to impedance measurements and spectroscopy analysis [10]. The low-pass filter corner was set at approximately $1 \mathrm{MHz}$ frequency, with $R_{2}$ and $C_{2}$ circuit elements, while the high-pass filter corner at $100 \mathrm{~Hz}$, with $G_{m h p}$ and $C_{1}$ components for its implementation. Special care was given to common-mode rejection ratio derived from possible electrode mismatch, input noise performance and low-power supply consumption. The frequency response, magnitude and phase are illustrated in Fig. 4, for an input voltage of amplitude $10 \mathrm{mV}$ and $\alpha_{i a}=10$. It can be observed how phase response is not constant at the passband, increasing phase errors at the edges. This additive effect can be corrected by calibration. Also, gain is not constant at bandpass edges, which could decrease the closed-loop gain $\left(\alpha_{o}\right)$ and increase the errors at impedance magnitude.

\subsection{The rectifier and error amplifier}

The full wave rectifier in Fig. 5a was developed by the authors, and it is based on pass transistors (MP, MN) to load the capacitor $C_{r}$ at the nearest voltage of $V_{o}$. The two comparators detect when the input signal is higher (lower) than $V_{o p}\left(V_{o m}\right)$ in each instant, charging then the $C_{r}$ capacitors to the $V_{o}$ peak (positive or negative) value. Settling time for comparators must be small for fast voltage input resolution. The discharge of $C_{r}$ is done by current sources, $I_{\text {dis }}$, and has been set to $1 \mathrm{mV}$ voltage ripple in a time period. Fig. 5b illustrates the waveforms obtained by electrical simulations for the upper and lower rectified signals at $10 \mathrm{kHz}$, for $C_{r}=20 \mathrm{pF}, I_{\text {dis }}=200 \mathrm{pA}$. In spectroscopy analysis, when frequency changes in a given range, the discharge current should be programmed at each frequency to fulfil the estimated $1 \mathrm{mV}$ voltage ripple in the rectifier output voltage. At the output voltages, $V_{\text {om }}$ and $V_{\text {op }}$, a differential-to-single amplifier [14] was added for single rail conversion. Its output, $V_{d c}$, has a gain $\alpha_{d c}$ respect to rectifier input amplitude, $V_{o}$.

To compare the rectifier output voltage $V_{d c}$ with $V_{r e f}$, the difference is amplified, creating the magnitude voltage signal, $V_{m}$, which has information on impedance magnitude. A simple twostage operational amplifier, in open loop configuration, is employed for error amplification [14]. The voltage signal $V_{d c}$ must be as near as possible to $V_{r e f}$.

\subsection{The current controlled circuit}

For $i_{x}$ amplitude programming, a four-quadrant multiplier and an OTA were designed. Both are placed in series as shown in Fig. 6.

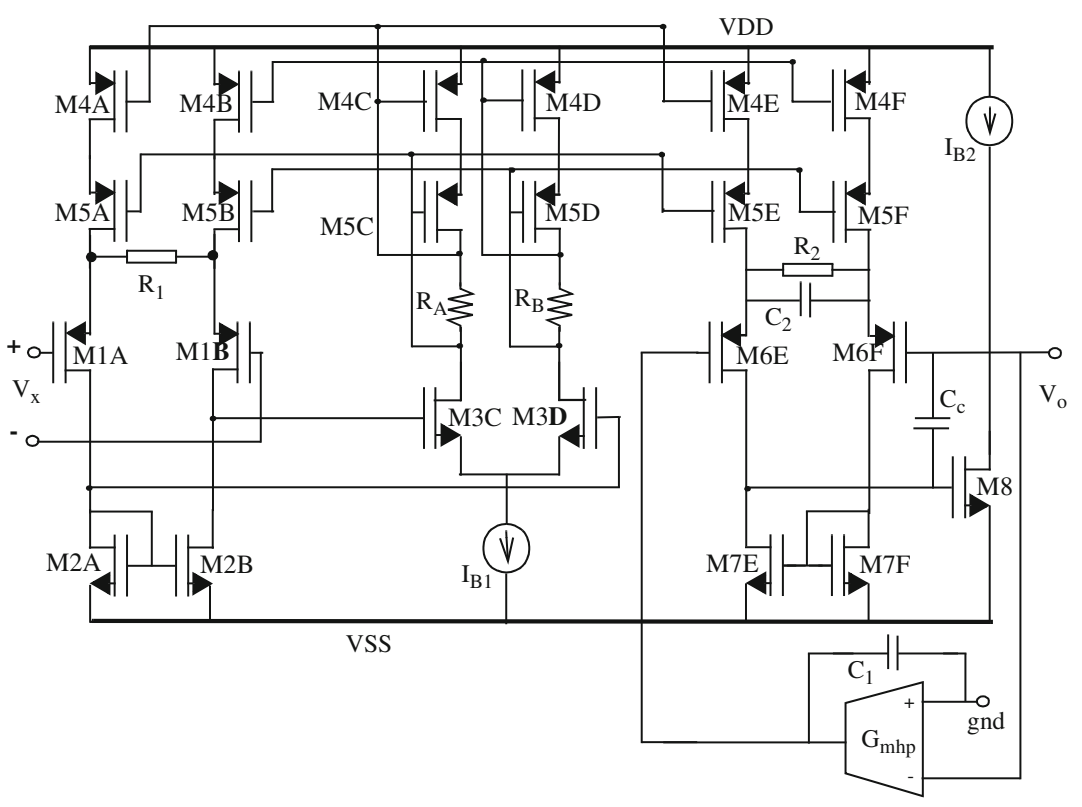

Transistor sizes $(\mu \mathrm{m} / \mu \mathrm{m})$ and components

\begin{tabular}{|c|c|}
\hline M1A-B & $200 / 2$ \\
\hline M2A-B & $10 / 10$ \\
\hline M3C-D & $100 / 2$ \\
\hline M4A-F & $100 / 1.5$ \\
\hline M5A-F & $20 / 1.5$ \\
\hline M6E-F & $200 / 2$ \\
\hline M7E-F & $100 / 2$ \\
\hline $\mathrm{M} 8$ & $66 / 1$ \\
\hline $\mathrm{G}_{\mathrm{mh}}$ & $200 \mathrm{nS}$ \\
\hline $\mathrm{C}_{1}$ & $15.9 \mathrm{pF}$ \\
\hline $\mathrm{C}_{2}$ & $1.9 \mathrm{pF}$ \\
\hline $\mathrm{R}_{1}$ & $1 \mathrm{k} \Omega$ \\
\hline $\mathrm{R}_{2}$ & $10 \mathrm{k} \Omega$ \\
\hline $\mathrm{Cc}_{\mathrm{c}}$ & $5 \mathrm{pF}$ \\
\hline $\mathrm{I}_{\mathrm{B} 1}$ & $70 \mu \mathrm{A}$ \\
\hline $\mathrm{I}_{\mathrm{B} 2}$ & $40 \mu \mathrm{A}$ \\
\hline
\end{tabular}

Fig. 3. CMOS instrumentation amplifier schematic. 


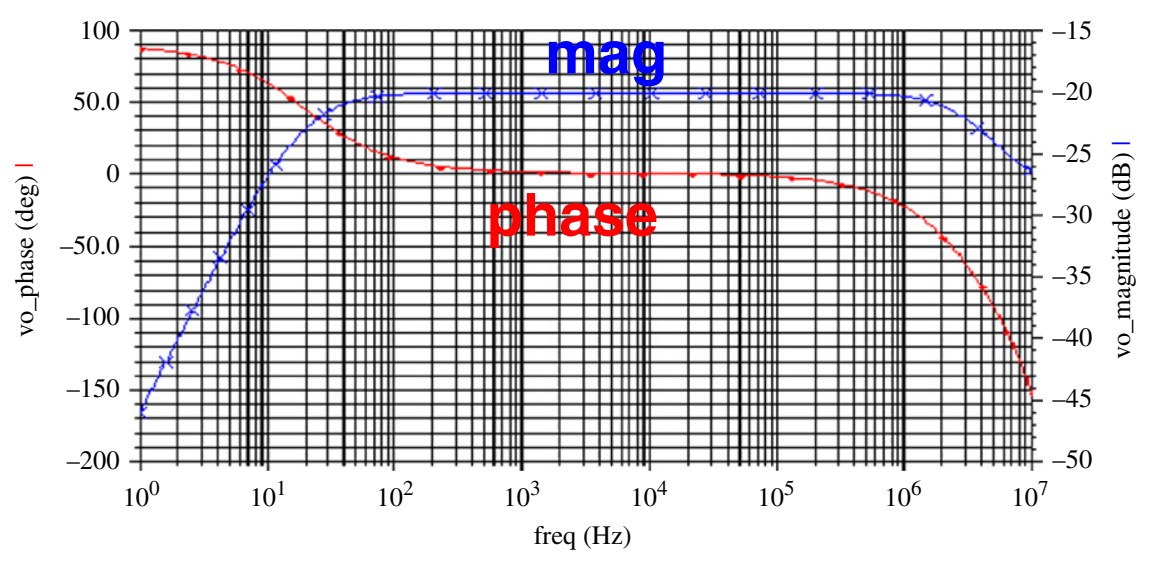

Fig. 4. Instrumentation amplifier magnitude and phase frequency response for $10 \mathrm{mV}$ input amplitude.

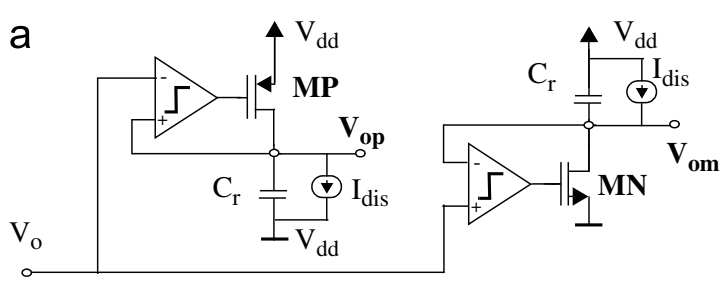

b

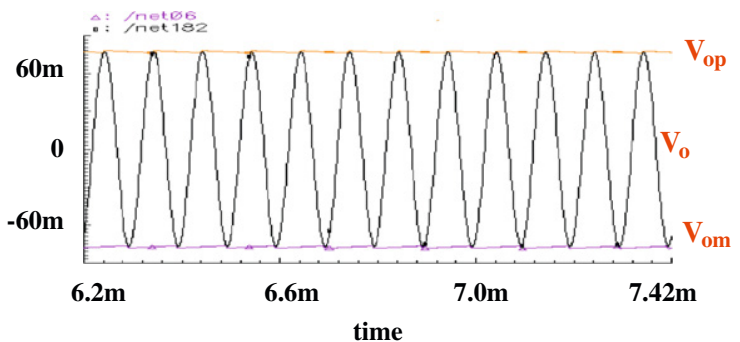

Transistor sizes $(\mu \mathrm{m} / \mu \mathrm{m})$ and components

\begin{tabular}{|c|c|}
\hline $\mathrm{MP}$ & $1 / 20$ \\
\hline $\mathrm{MN}$ & $1 / 40$ \\
\hline $\mathrm{C}_{\mathrm{r}}$ & $20 \mathrm{pF}$ \\
\hline $\mathrm{I}_{\mathrm{dis}}$ & $200 \mathrm{pA} @ 10 \mathrm{kHz}$ \\
\hline
\end{tabular}

C

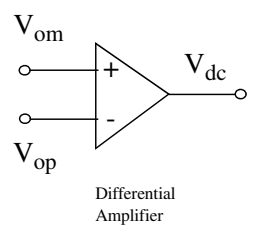

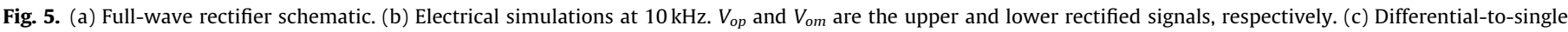
voltage amplifier.

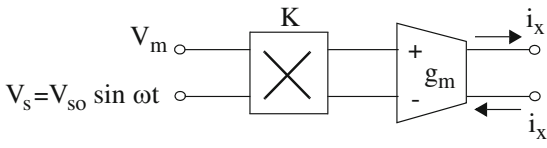

Fig. 6. Blocks of the current controlled circuit: a multiplier in series with an OTA.

The external AC voltage source is first multiplied by the voltage $V_{m}$. The result is later converted to AC current for load excitation.

The schematic of the four-quadrant multiplier circuit is shown in Fig. 7 [11]; this was selected because inputs are AC signals, its topology is simple, it has reduced number of transistors and can work at low-voltage low-power conditions. It has two inputs: the external AC voltage generator, $V_{s}$, and the voltage magnitude, $V_{m}$. The multiplier output waveforms are shown in Fig. 8. In this figure, the AC signal $V_{s}$ has $200 \mathrm{mV}$ of amplitude at $10 \mathrm{kHz}$ frequency, and it is multiplied by a DC signal, $V_{m}$, in the range $[0,200 \mathrm{mV}]$. The differential output is given by

$V_{\text {out }}=V_{\text {out } 1}-V_{\text {out } 2}=2 R \sqrt{k_{n} k_{p}} V_{m} V_{s}=K V_{m} V_{s}$

where $K$ is the constant of the multiplier, and $k_{n}$ and $k_{p}$ the transconductance parameters for M1 and M6 transistors.
The operational transconductance amplifier employed has the schematic in Fig. 9 [10]. The cascode output stage reduces the load effect due to large ohmic values in loads $\left(Z_{x o}\right)$. Typical output resistance for cascode output stages is greater than $100 \mathrm{M} \Omega$, so errors expected due to load resistance effects will be small.

\subsection{The comparator}

The voltage comparator selected is shown in Fig. 10 [14]. A chain of inverters have been added at its output for fast response and regeneration of digital levels. The comparator is employed both for rectifying the AC signal and squaring the sinusoidal voltage signals for obtaining full range digital signal at the EXOR gate inputs.

From the design data an amplitude is obtained for the voltage $V_{x}$ of $8 \mathrm{mV}$ over the load $Z_{x}$. In electrode-based measurements, $V_{x o}$ has typically low and limited values (tens of $\mathrm{mV}$ ) to control its expected electrical performance [5]. This condition is preserved by design, thanks to the voltage limitation imposed by the Pstat operation mode, and can be tuned by changing the value of $V_{\text {ref }}$ (in our case $20 \mathrm{mV}$ ), as it is derived from Eq. (1). 


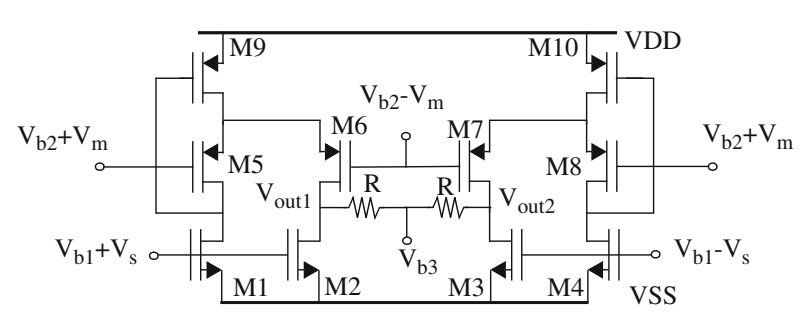

Transistor sizes $(\mu \mathrm{m} / \mu \mathrm{m})$ and components

Fig. 7. Multiplier circuit. $V_{b 1}, V_{b 2}$ and $V_{b 3}$ are bias voltages.

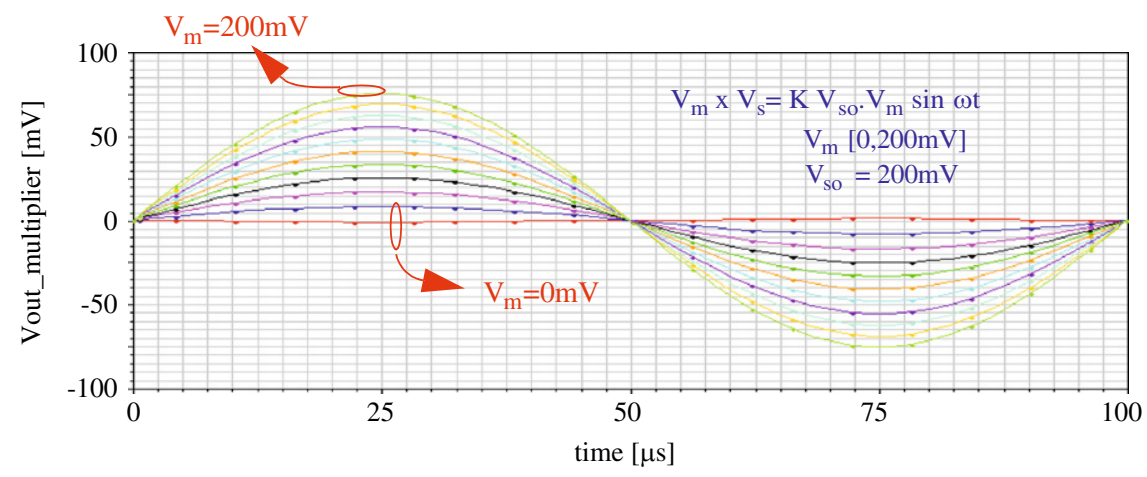

Fig. 8. Simulated multiplier output voltage waveforms.

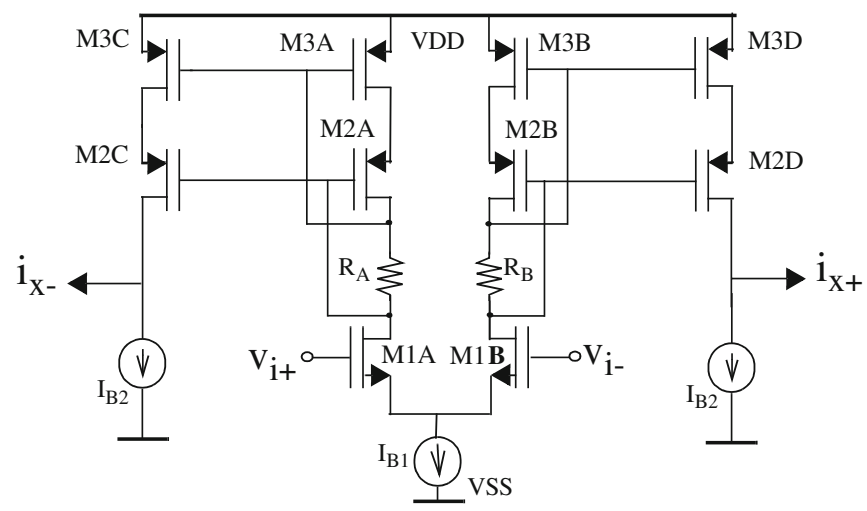

Transistors sizes $(\mu \mathrm{m} / \mu \mathrm{m})$ and components

\begin{tabular}{|c|c|}
\hline $\mathrm{M} 1 \mathrm{~A}-\mathrm{B}$ & $5 / 5$ \\
\hline $\mathrm{M} 2 \mathrm{~A}-\mathrm{D}$ & $20 / 1.5$ \\
\hline $\mathrm{M} 3 \mathrm{~A}-\mathrm{D}$ & $20 / 1.5$ \\
\hline $\mathrm{R}_{\mathrm{A}}, \mathrm{R}_{\mathrm{B}}$ & $20 \mathrm{k} \Omega$ \\
\hline $\mathrm{I}_{\mathrm{B} 1}$ & $0.530 \mu \mathrm{A}$ \\
\hline $\mathrm{I}_{\mathrm{B} 2}$ & $0.265 \mu \mathrm{A}$ \\
\hline
\end{tabular}

Fig. 9. OTA circuit schematic

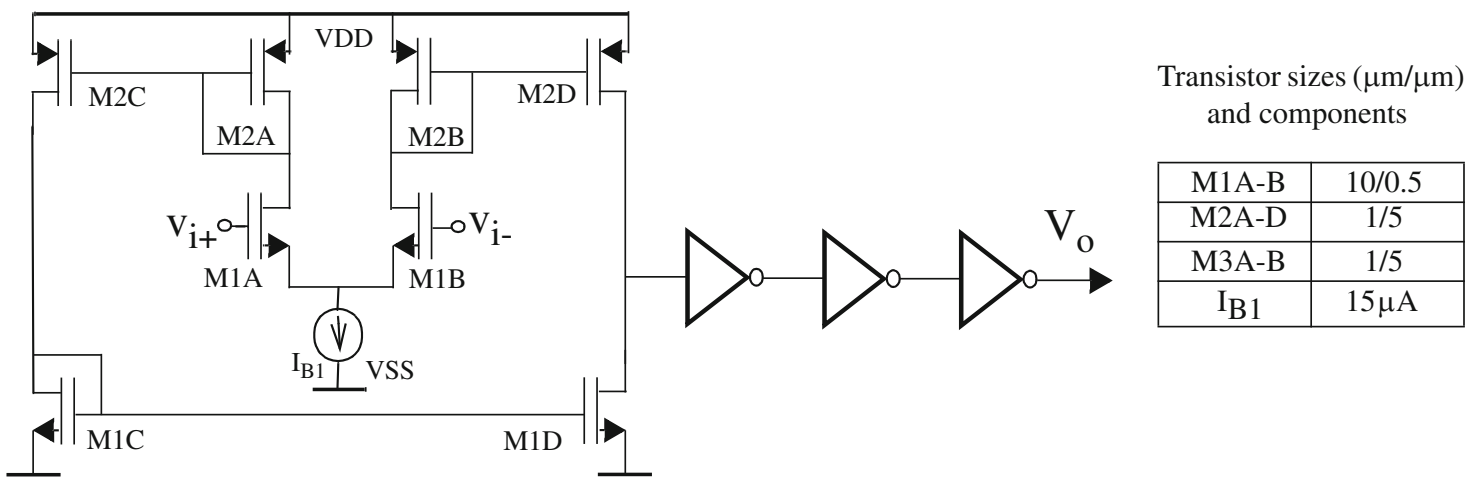

Fig. 10. Comparator circuit schematic.

\section{System design considerations}

Due to the high loop gain for satisfying condition in Eq. (2), it is necessary to study the stability of the system. In steady-state, eventual changes produced at the load can generate variations at the rectifier output voltage, which will be amplified $\alpha_{e a}$ times, leading to out of range for some circuits. To avoid this, some control mechanism should be included in the loop. A first order low-pass filter was selected at the error amplifier output. This LPF in Fig. 11 acts as a delay element, avoiding an excessive fast 


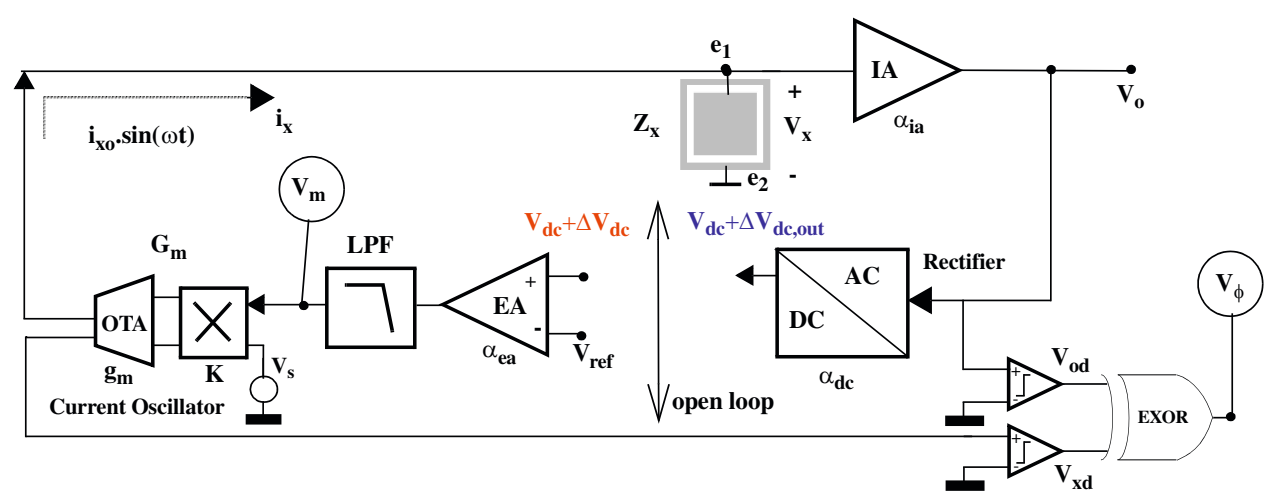

Fig. 11. Open loop system for steady-state stability analysis.

a

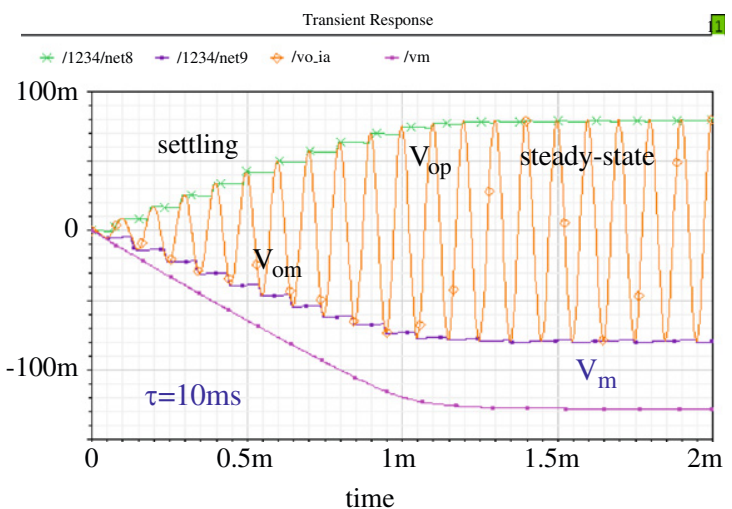

b

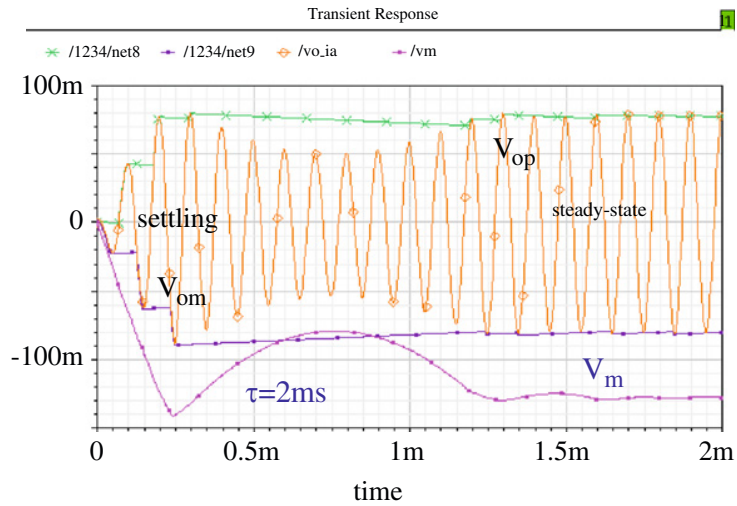

Fig. 12. Start-up transient from $V_{m}=0$ to its steady-state, for (a) $\tau=10 \mathrm{~ms}$ and (b) $\tau=2 \mathrm{~ms}$, at $f=10 \mathrm{kHz}$.

response in the loop, by setting a dominant pole. For a given $\Delta V_{d c}$ voltage increment, in a period of time of the AC signal, the gain of the loop must be below unity. To analyse the response of the loop to a $\Delta V_{d c}(t)$ voltage increment, we cut the loop between the rectifier and error amplifier. The corresponding voltage response will be

$\Delta V_{d c, \text { out }}=Z_{x o} G_{m} \alpha_{e a} \alpha_{i a} \alpha_{d c}\left(1-e^{-t / \tau}\right) \Delta V_{d c}$

For a gain below unity in a period of time $t=\mathrm{T}$, the output voltage increment of the rectified signal must be lesser than the corresponding input voltage changes, $\Delta V_{d c \text {,out }}<\Delta V_{d c}$; hence,

$1<Z_{x o} G_{m} \alpha_{e a} \alpha_{i a} \alpha_{d c}\left(1-e^{-t / \tau}\right)$

which means an LPF time constant given by

$\tau>\frac{T}{\ln \left(\frac{\alpha_{o}}{\alpha_{0}-1}\right)}$

This condition makes the filter design dependent on ZUT through the parameter $Z_{x o}$, which should be quoted in order to apply the condition in Eq. (7) properly. For example, if we take $\alpha_{o}$ $=100$, for a $10 \mathrm{kHz}$ working frequency, the period is $T=0.1 \mathrm{~ms}$, and $\tau<9.94991 \mathrm{~ms}$. If $\tau=R_{F} \cdot C_{F}$ in a discrete first order filter realization, for $C_{F}=20 \mathrm{pF}$, it corresponds to $R_{F}=500 \mathrm{M} \Omega$. Preserving by design large $\alpha_{o}$ values, imposed by Eq. (2), the working frequency will define the values of $\tau$ in LPF. For load dependent values on $\alpha_{o}$, as the case of living cells on top of electrodes, Eq. (7) should consider the biggest value of $\alpha_{o}$ for quoting the worst case design. The stability problem is also present at the start-up operation, for each new measure. In this situation, reset is applied to the system by initializing to zero the filter capacitor. Measurement process starts from $V_{m}=0$, and after several periods of time it is found to achieve steady-state. This is the time required to load the capacitors $C_{r}$, at rectifier, up to their steadystate value. This can be observed at the waveforms in Fig. 12, for $\tau=10 \mathrm{~ms}$ and $\tau=2 \mathrm{~ms}$, where the settling transient at the rectifier envelops are also represented. When signal $V_{o}$ (IA output) found the value of $80 \mathrm{mV}$, the loop starts working. In the first case ( $\tau=10 \mathrm{~ms}$ ), is satisfied condition in Eq. (7). It can be observed how if $\tau=2 \mathrm{~ms}$, transient to final value is not well controlled. The number of periods required for settling is $N_{c}$ and the time required to perform a measure is defined as T.N. $N_{c}$. The influence of the input noise of the instrumentation amplifier over the output signal $\left(V_{m}\right)$ will be reduced as a consequence of this LP filtering process.

\section{Simulation results}

A first set of electrical simulations was performed at $10 \mathrm{kHz}$ frequency, using for $Z_{x}$ resistive $(100 \mathrm{k} \Omega)$, RC $(100 \mathrm{k} \Omega \| 159 \mathrm{pF})$ and capacitive $(159 \mathrm{pF})$ loads. Fig. 13 shows the waveforms obtained using spectre electrical simulator for excitation signals, $V_{x}$ and $i_{x}$, and the resulting signals derived to obtain magnitude, $V_{m}$, and phase, $V_{\phi}$. The amplifier output voltage $V_{o}$ is nearly constant and equal to $80 \mathrm{mV}$ for all loads, fulfilling the Pstat condition $\left(V_{x o}=V_{o}\right)$ $\alpha_{a i}=8 \mathrm{mV}$ ), while $i_{x}$ has an amplitude adapted to load for each case. The $V_{m}$ value gives the expected magnitude of $Z_{x o}$ using Eqs. (1) and (3) in all cases, as shown in Table 1 . The measure duty cycle allows the calculus of the $Z_{x}$ phase. The $10 \mathrm{kHz}$ frequency has been selected because the phase shift introduced by the instrumentation amplifier is close to zero, minimizing its influence on phase calculations. This and other deviations from ideal performance derived from process parameters variations 

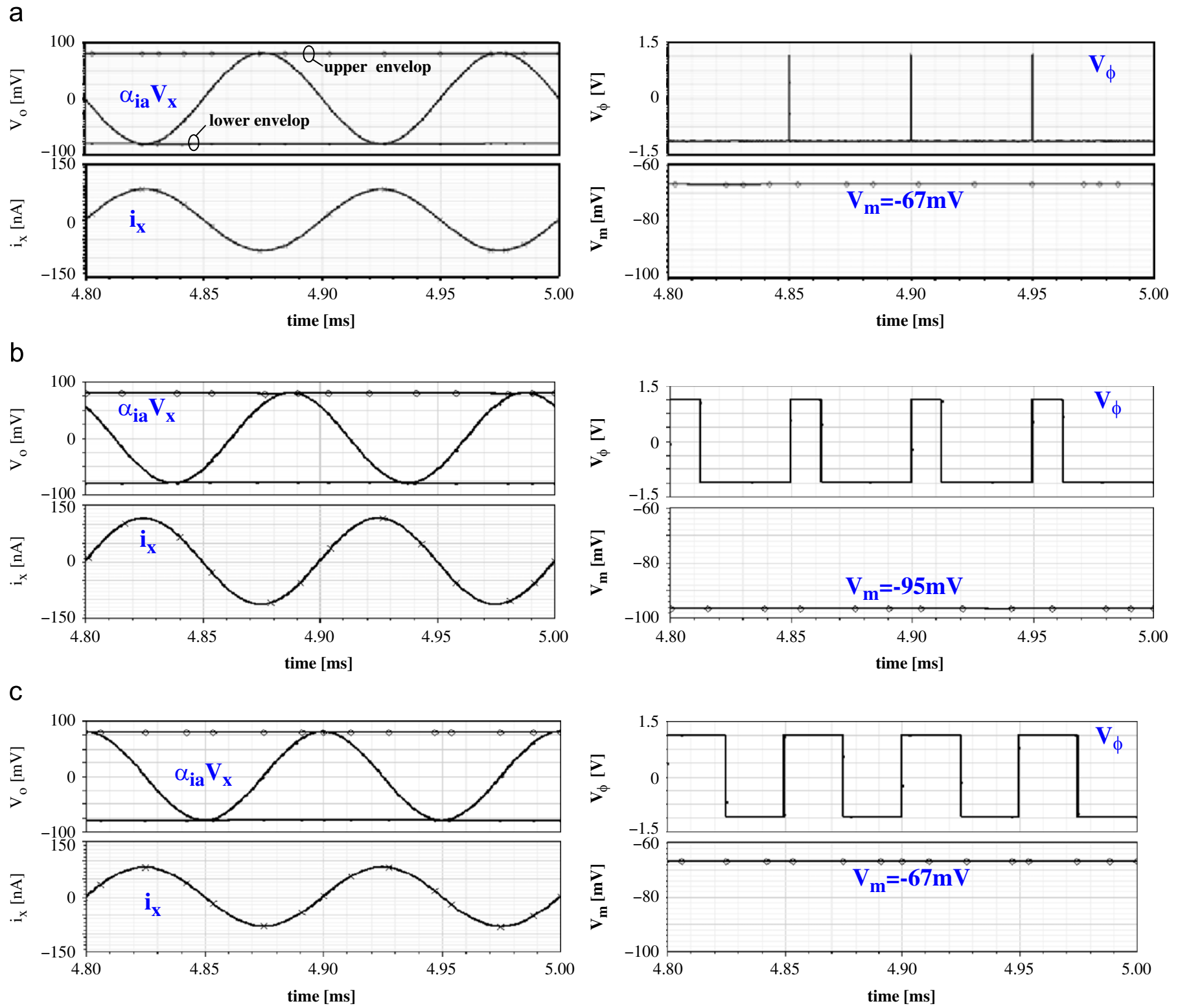

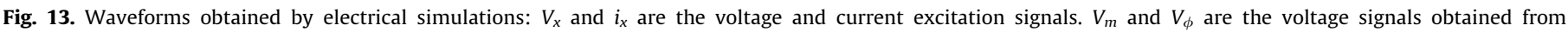
measurement circuits using (a) $R_{x}=100 \mathrm{k} \Omega$. (b) $R_{x} C_{x}=(100 \mathrm{k} \Omega \| 159 \mathrm{pF})$ and (c) $C_{x}=159 \mathrm{pF}$.

Table 1

Simulation results at $10 \mathrm{kHz}$ for several $Z_{x}$ loads.

\begin{tabular}{|c|c|c|c|c|c|c|}
\hline \multirow[t]{2}{*}{$Z_{x}$} & \multirow{2}{*}{$\begin{array}{l}\mathrm{V}_{m}[\mathrm{mV}] \\
\operatorname{sim}\end{array}$} & \multirow{2}{*}{$\begin{array}{l}\delta \\
\operatorname{sim}\end{array}$} & \multicolumn{2}{|c|}{$\mathrm{Z}_{x o}[\mathrm{k} \Omega]$} & \multicolumn{2}{|l|}{$\phi\left[^{\circ}\right]$} \\
\hline & & & $\operatorname{sim}$ & teo & $\operatorname{sim}$ & teo \\
\hline case $\mathrm{R}$ & 67.15 & 0.005 & 99.28 & 100.0 & 00.93 & 0 \\
\hline case RC & 94.96 & 0.247 & 70.20 & 70.70 & 44.44 & 45 \\
\hline case $C$ & 67.20 & 0.501 & 99.21 & 100.0 & 90.04 & 90 \\
\hline
\end{tabular}

should be adjusted by calibration. Errors in both parameters are within the expected range (less than $1 \%$ ).

Another parallel RC load has been simulated. In this case, the working frequency is $100 \mathrm{kHz}, C_{x}=15.9 \mathrm{pF}$, and the values of $R_{x}$ are in the range [ $10 \mathrm{k} \Omega, 1 \mathrm{M} \Omega$ ]. The results are listed in Table 2 and represented in Fig. 14. Excellent agreement was obtained for both magnitude and phase with the theory.

A four-wire system for $Z_{x}$ measurements is shown in Fig. 15a. This kind of set-up can be useful in electrical impedance
Table 2

Simulation results for $R_{x} \| C_{x}$ load. $\left(C_{x}=15.9 \mathrm{pF}, f=100 \mathrm{kHz}, \phi_{I A}(100 \mathrm{kHz})=2.3^{\circ}\right.$, $G_{m}=1.6 \mu \mathrm{S}$

\begin{tabular}{llllrc}
\hline$R_{x}[\mathrm{k} \Omega]$ & $V_{m}[\mathrm{mV}]$ & $\delta[\mathrm{us}]$ & $V_{x o}[\mathrm{mV}]$ & $Z_{x o}[\mathrm{k} \Omega]$ & $\phi\left[{ }^{\circ}\right]$ \\
\hline 10 & 491.0 & 0.24 & 7.8 & 9.92 & 6.34 \\
20 & 251.2 & 0.40 & 7.8 & 19.43 & 12.1 \\
50 & 112.7 & 0.83 & 7.9 & 43.60 & 27.6 \\
100 & 69.7 & 1.34 & 7.9 & 70.80 & 43.9 \\
200 & 55.2 & 1.85 & 7.9 & 89.53 & 64.3 \\
500 & 50.4 & 2.27 & 7.9 & 97.97 & 79.4 \\
1000 & 49.7 & 2.42 & 7.9 & 99.35 & 84.8 \\
\hline
\end{tabular}

tomography (EIT) of a given object [7], decreasing the electrode impedance influence $\left(Z_{e 1}\right.$ to $\left.Z_{e 4}\right)$ on the output voltage $\left(V_{o}\right)$, thanks to the instrumentation amplifier high input impedance. The model used for the electrode in Fig. 15c considers the double layer capacitance $\left(C_{p}\right)$, the charge transfer resistance $\left(R_{p}\right)$ and the spreading resistance $\left(R_{s}\right)$ of an electrode-solution system [5]. 

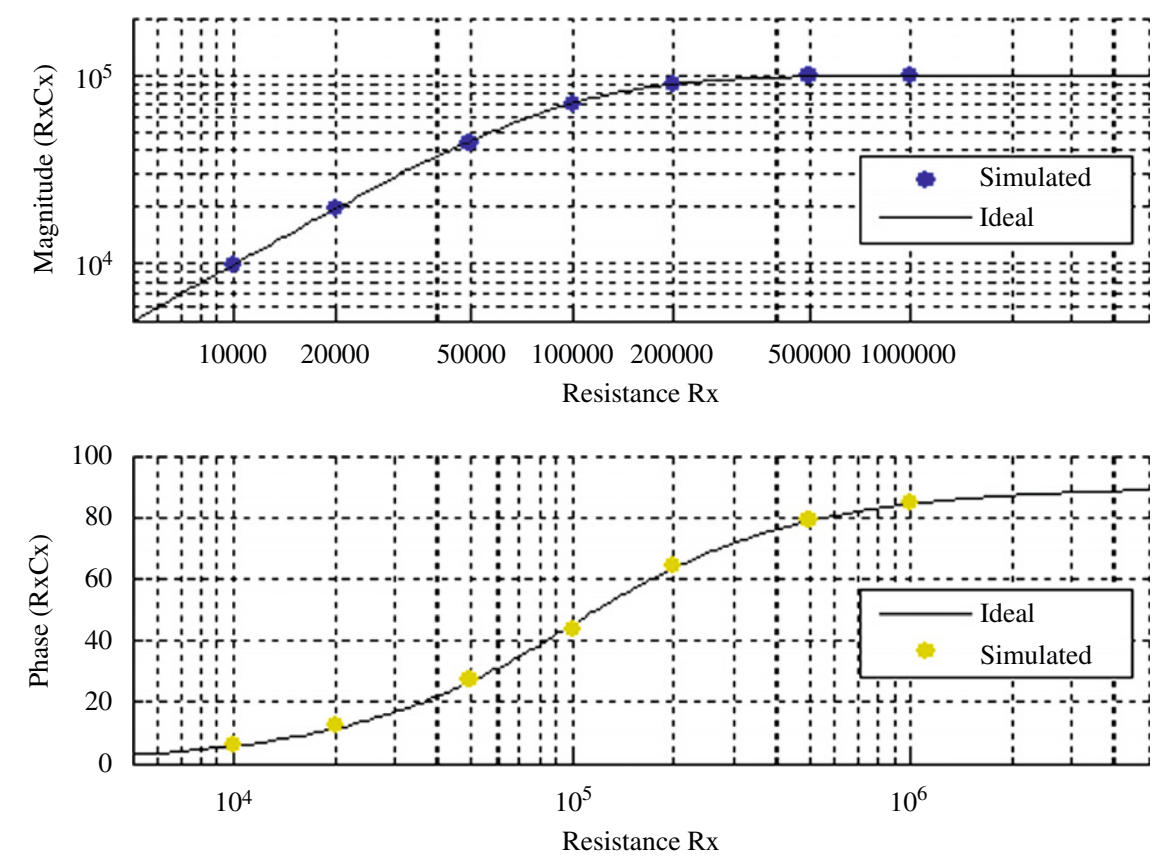

Fig. 14. Magnitude and phase obtained from electrical simulations for $R_{x} \| C_{x}$ using $C_{x}=15.9 \mathrm{pF}$ and $R_{x}$ belongs to [10 k $\left.2,1 \mathrm{M} \Omega\right]$ at $f=100 \mathrm{kHz}$.

a

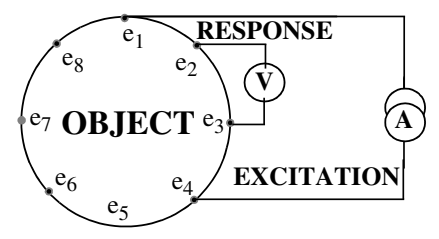

b

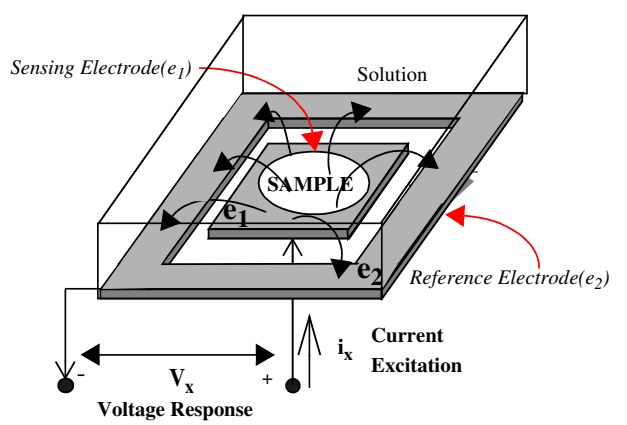

C

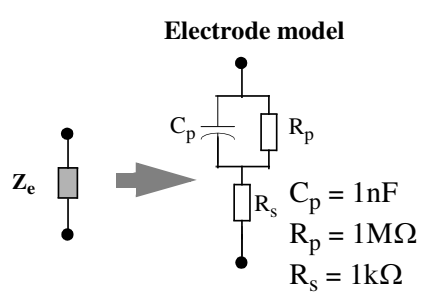

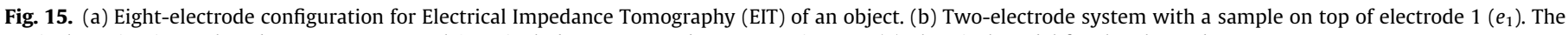
equivalent circuit employed uses $R_{\mathrm{SAMPLE}}=100 \mathrm{k} \Omega$. $Z_{x}$ includes $Z_{e 1}, Z_{e 2}$ and $R_{\mathrm{SAMPLE}}$ resistance. (c) Electrical model for the electrode.

Table 3

Simulation results for four-electrode setup and a load $Z_{x}=100 \mathrm{k} \Omega$.

\begin{tabular}{llrlrr}
\hline frequency $[\mathrm{kHz}]$ & \multicolumn{2}{l}{$Z_{x o}[\mathrm{k} \Omega]$} & & \multicolumn{2}{l}{$\phi\left[^{\circ}\right]$} \\
\cline { 2 - 3 } \cline { 5 - 6 } & sim & teo & & \multicolumn{1}{c}{$\operatorname{sim}$} & \multicolumn{1}{c}{ teo } \\
\hline 0.1 & 96.17 & 92.49 & & 11.70 & 13.67 \\
1 & 99.40 & 100.00 & & 1.22 & 1.90 \\
10 & 99.80 & 100.00 & & -0.20 & -0.12 \\
100 & 99.70 & 100.00 & & -4.10 & -3.20 \\
1000 & 95.60 & 96.85 & & -40.60 & -32.32 \\
\hline
\end{tabular}

Parameters values have been taken from [3]. Using a $100 \mathrm{k} \Omega$ load at $10 \mathrm{kHz}$ frequency, the voltage at $Z_{x}$ load matches the amplitude of $V_{x o}=8 \mathrm{mV}$, and the calculus of the impedance value at $10 \mathrm{kHz}$ frequency $\left(Z_{x o}=99.8 \mathrm{k} \Omega\right.$ and $\left.\phi=0.2^{\circ}\right)$ is correct. The same load is maintained in a wide range of frequencies $(100 \mathrm{~Hz}$ to $1 \mathrm{MHz}$ ), achieving the magnitude and phase listed in Table 3. The main deviations are present at the amplifier bandpass frequency edges due to lower and upper $-3 \mathrm{~dB}$ frequency corners. It can be observed the phase response measured and the influence due to amplifier frequency response in Fig. 4. In this case, for spectroscopy analysis, discharge current, $I_{d i s}$, in full wave rectifier has been selected to fulfil $1 \mathrm{mV}$ output voltage ripple. In a similar way, the equivalent transconductance $G_{m}$ and the time constant at LPF in Fig. 11 should be programmed to each working frequency.

A two-electrode system is employed in Electric Cell substrate Impedance Spectroscopy (ECIS) [6] as a technique capable of obtaining basic information on single or low concentration of cells. The main drawback of two-wire systems is the output signal corresponds to the series of two electrodes and the load, being necessary to extract the load from the measures [12,13]. Fig. 15b shows a two-electrode set-up in which the load or sample $(100 \mathrm{k} \Omega)$ has been measured in the frequency range of $[100 \mathrm{~Hz}$, $1 \mathrm{MHz}$. Circuit parameters were adapted to satisfy the $\alpha_{o}=100$ condition, since $Z_{x o}$ will change from around $1 \mathrm{M} \Omega$ to $100 \mathrm{k} \Omega$ when passing from tens of $\mathrm{Hz}$ to $\mathrm{MHz}$ frequencies, due to electrode impedance dependence. The simulation data obtained are shown in Table 4 . At $10 \mathrm{kHz}$ frequency, magnitude $Z_{x o}$ becomes $107.16 \mathrm{k} \Omega$, because it includes two electrodes in series. The same effect occurs for phase, now becoming $17.24^{\circ}$. Results are shown in Table 4 for the frequency range considered. The accuracy observed is better at the mid-bandwidth. Again, errors in magnitude and phase increase at the bandpass corners of the instrumentation amplifier. 
Table 4

Simulation results for two-electrode setup and a load $Z_{x}=100 \mathrm{k} \Omega$.

\begin{tabular}{lccccr}
\hline frequency $[\mathrm{kHz}]$ & \multicolumn{2}{l}{$Z_{x o}[\mathrm{k} \Omega]$} & & \multicolumn{2}{l}{$\phi\left[^{\circ}\right]$} \\
\cline { 2 - 3 } \cline { 5 - 6 } & $\operatorname{sim}$ & teo & & sim & teo \\
\hline 0.1 & 1058.8 & 1087.8 & & -40.21 & -19.00 \\
1 & 339.35 & 344.70 & & -56.00 & -62.88 \\
10 & 107.16 & 107.33 & & -17.24 & -17.01 \\
100 & 104.80 & 102.01 & & -6.48 & -5.09 \\
1000 & 104.24 & 102.00 & & -37.80 & -32.24 \\
\hline
\end{tabular}

In both cases, when using electrodes, the equivalent circuit described in [3] was employed for the electrode model. This circuit represents a possible and real electrical performance of electrodes in some cases. In general, the electric model for electrodes will depend on the electrode-to-sample [12,13] and/or medium [5] interfaces and should be adjusted to each test problem. In this work, a real electrical electrode model was used to confirm the adequate performance of the proposed CMOS measurement system.

\section{Conclusions}

This paper proposes the design of a CMOS system for impedance measurement, useful for sensing biological samples, thanks to the possibility of including the electrode specifications at the design process. The proposed system works using Pstat condition to control the voltage amplitude on the sensing electrodes, and can be designed to work at several frequencies. New feedback circuit implementations for both exciting the load and delivering measure signals are proposed. Input offset and noise amplifier are attenuated, thanks to high-pass and low-pass filter functions, respectively. Design trade-offs for system accuracy and stability have been derived. Electrical simulations prove the correct circuit performance, obtaining magnitude and phase errors below $1 \%$ for several frequencies and measurement setups.

\section{Acknowledgement}

This work was in part supported by the Spanish founded Project: TEC2007-68072, Técnicas para mejorar la calidad del test y las prestaciones del diseño en tecnologías CMOS submicrométricas.

\section{References}

[1] J.J. Ackmann, Complex bioelectric impedance measurement system for the frequency range from 5-1 MHz, Annals of Biomedical Engineering 21 (1993) $135-146$.

[2] R.D. Beach, R.W. Conlan, M.C. Godwin, Moussy Francis, Towards a miniature in vivo telemetry monitoring system dynamically configurable as a potentiostat or galvanostat for two- and three-electrode biosensors, IEEE Transactions on Instrumentation and Measurement 54 (1) (2005) 61-72.

[3] A. Yœfera, A. Rueda, J.M. Mu-oz, R. Doldłn, G. Leger, E.O. Rodr’guez-Villegas, A Tissue impedance measurement chip for myocardial ischemia detection, IEEE Transaction on Circuits and Systems: part I 52 (12) (2005) 2620-2628.

[4] S.M. Radke, E.C. Alocilja, Design fabrication of a microimpedance biosensor for bacterial detection, IEEE Sensor Journal 4 (4) (2004) 434-440.

[5] D.A. Borkholder, Cell-based biosensors using microelectrodes, Ph.D. Thesis, Stanford University, November 1998.

[6] I. Giaever, C.R. Keese, Use of electric fields to monitor the dynamical aspect of cell behaviour in tissue culture, IEEE Transactions on Biomedical Engineering, BME 33 (2) (1986) 242-247.

[7] D. Holder, Electrical impedance tomoghaphy: methods, history and applications, Philadelphia: IOP, 2005.

[8] R. Pall\$s, J.G. Webster, Bioelectric impedance measurements using synchronous sampling, IEEE Transactions on Biomedical Engineering 40 (8) (1993) 824-829.

[9] A. Yœfera, A. Rueda, A method for bioimpedance measure with four- and twoelectrode sensor systems, 30th Annual International IEEE EMBS Conference (2008) 2318-2321.

[10] Y.-Q. Zhao, A. Demosthenous, R.H. Bayford, A CMOS instrumentation amplifier for wideband bioimpedance spectroscopy systems, International Symposium on Circuits and Systems (ISCAS) (2006) 5079-5082.

[11] C. Sawigun, A Demosthenous Compact low-voltage CMOS four-quadrant analogue multiplier, Electronics Letters 42 (20) (2006).

[12] X. Huang, D. Nguyen, D.W. Greve, M. Domach, Simulation of microelectrode impedance changes due to cell growth, IEEE Sensors Journal 4 (5) (2004) 576-583.

[13] N. Joye, A. Schmid, Y. Leblebici, An electrical model of the cell-electrode interface for high-density microelectrode arrays, 30th Annual International IEEE EMBS Conference (2008) 559-562.

[14] P.E. Allen, D.R. Holberg, CMOS Analog Circuit Design, 2nd Ed., Oxford University Press, 2002 